\title{
European SWaption by Monte Carlo Estimation on MODERN INTEL ARCHITECTURES
}

\author{
Eugen Mudnic, Stipe Celar, Zeljko Seremet, Filipa Matic
}
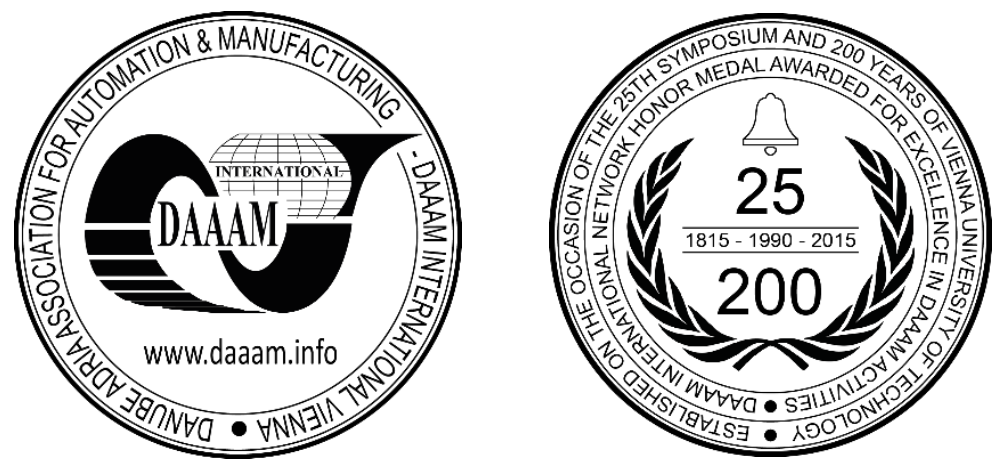

This Publication has to be referred as: Mudnic, E[ugen]; Celar, S[tipe]; Seremet, Z[eljko] \& Matic, F[ilipa] (2017). European Swaption by Monte Carlo Estimation on Modern Intel Architectures, Proceedings of the 28th DAAAM International Symposium, pp.0351-0356, B. Katalinic (Ed.), Published by DAAAM International, ISBN 978-3-90273411-2, ISSN 1726-9679, Vienna, Austria

DOI: $10.2507 / 28$ th.daaam.proceedings.048

\begin{abstract}
Modern computing resources provide substantial processing power but require that the programmatic implementation of used numerical algorithms is fine-tuned to the target CPU architecture. The main performance gains could be obtained minimizing cache traffic and by efficient using of multiple levels of CPU parallel units. Monte Carlo estimation of LIBOR models is an example of challenging computational finance/computer programming problem. This paper compares the efficiency of multicore (Intel Core Haswell) and manycore platforms (Intel Xeon Phi Knight Corner and Intel Xeon Phi Knight Landing) for the calculation European LIBOR-based swaptions. Target optimized programmatic implementations of LIBOR calculation using the Intel Cilk Plus and OpenMP standards are presented and benchmarked. Results show that Intel Xeon Phi Knight Landing evaluate payoff European LIBOR-based swaptions faster, economically profitable and more energy efficient than the Intel Xeon Phi Knight Corner coprocessor and Intel Core Haswell.
\end{abstract}

Keywords: Libor; Monte Carlo simulation; Intel Cilk Plus; OpenMP; Intel Knights Landing

\section{Introduction}

The London interbank offered rate, also called LIBOR [1] is one of the main instruments in the debt market and it is the basis for many derived interest rate instruments. Along with numerous analytical methods, "brute force" Monte Carlo approach could be used to simulate prices of LIBOR-based swaptions [1]. Numerous papers [2][3][4][5][6][7][8][9][10][11] confirm that the Monte Carlo prices of LIBOR-based swaptions could not be calculated in a reasonable time without high level of parallelization. Currently Intel processors are evolving from multicore (Intel Core) toward manycore (Intel Xeon Phi) architectures, offering massive parallelization capabilities even on single CPU. Although the declared theoretical peak power (GFLOPs) show significant performance gain for manycore processors, the economic performance-per-price and performance-per-watt are still questionable. The purpose of the present work is therefore to first efficiently tune parallelization of the LIBOR Monte Carlo calculation both on multicore and manycore CPUs, and then to compare both their execution times and main economic performance.

There are many LIBOR test cases with different Monte Carlo settings [12][13][14]. In this paper we used algorithm [15] based on an original code by Paul Glasserman and Xiaoliang Zhao [12][16][18] with subsequent modifications by 
Mike Giles [13][19]. The algorithm requires repeated simulations of chance that lend themselves well to parallel (thread) processing and vectorization on multicore and manycore Intel CPUs.

For writing the parallelization code, we used alternatively Intel Cilk Plus and OpenMP frameworks and Intel vectorization $\mathrm{C}++$ language extensions as available using the Intel Compiler v17.0. The used algorithm was optimized to the target CPU architecture regarding to differences in available instruction sets (AVX 2.0, IMCI, and AVX-512) between target architectures: Intel Xeon Haswell (HSW), Intel Xeon Knight Corner (KNC), and Intel Xeon Knight Landing (KNL). We evaluated the economic profitability and the energy efficiency using computation time in representative benchmarks. On these benchmarks, we demonstrate best performance and also economic gains on the KNL.

The paper is organized as follows. Section 2 describes used algorithm. Section 3 describes implementation details. Section 4 evaluates showing performance results on real benchmarks. Section 5 presents our conclusion and future work.

\section{Used algorithm}

Monte Carlo simulation is very usefully technique in capacity planning [17] and to compute the correct prices for financial options in computational finance especially for the calculation of LIBOR. In mathematical finance, we have two classic methods used to compute LIBOR in a Monte Carlo setting: the pathwise sensitivities and the Likelihood Ratio Method. In this section, we brief review of LIBOR market models based on a finite set of maturities, as developed by Jamshidian [18].

The forward LIBOR rate at time $t$ for the accrual period $\left[T_{i} ; T_{i+1}\right]$, with $t \leq T_{\mathrm{i}}$, is

$$
L_{i}(t)=\frac{1}{\delta}\left(\frac{B_{i}(t)}{B_{i+1}(t)}-1\right), i=1, \ldots, N
$$

It is at times notational convenient to extend the definition of $L_{i}$ beyond the $i$ th tenor date; we do so by setting $L_{i}(t)=$ $L_{i}\left(T_{i}\right)$ for $t<T_{i}$. At a tenor date $T_{i}$, the price of any bond $B_{n}$, with $\mathrm{n}>\mathrm{i}$, that has not yet matured is given by

$$
B_{n}\left(T_{i}\right)=\prod_{j=1}^{n-1} \frac{1}{1+\delta L_{j}\left(T_{i}\right)}
$$

more generally, at an arbitrary time $t<T_{n}$, we have

$$
B_{n}\left(T_{i}\right)=B_{\eta(t)}(t) \prod_{j=\eta(t)}^{n-1} \frac{1}{1+\delta L_{j}(t)}
$$

The time- $t$ value $C(t)$ and time- $T$ value $C(T)$ of a derivative security (that can be replicated by trading in the basic bonds) are related by the pricing rule

$$
C(t)=B^{*}(t) E\left[\frac{C(T)}{B^{*}(t)} \mid \mathcal{F}_{t}\right]
$$

where $\{\mathcal{F}, \mathrm{t} \geq 0\}$ is the filtration generated by the Brownian motion.

From (4) and the definition of $B^{*}$, it follows that its price at time 0 is

$$
\left.C_{n}(0)=B_{1}(0) \delta E\left[\left(L_{n}\left(T_{n}\right)\right)-K\right)^{+} \prod_{i=1}^{n} \frac{C(T)}{1+\delta L_{i}\left(T_{n}\right)}\right]
$$

See [16] for more details. The algorithm then proceeds as follows:

1. calculate Monte carlo paths,

2. calculate path for swaption kernel,

3. calculates the forward LIBOR rates over the time interval,

4. calculates the estimated strike price for the swaption,

5. discounts the forward LIBOR rate.

Each option in alghoritm step could be parellized using multiple threads or vectorization.

\section{Implementation Details}

Intel compiler $v 17.0$ offers support for different multithreading parallelization frameworks. We have used alternatively Intel Cilk Plus and OpenMP since they use very similar code extensions. Each of the parallel programming frameworks has their own internal implementation and it is expected different fit to problem we are solving. 


\subsection{Intel Cilk Plus}

Intel Cilk Plus [20] [21] is a powerful parallelization and vectorization framework that can effectively parallelize complex problems. In this framework, the programmer specifies the components of the application that can been run in parallel, and the runtime library takes care of assigning computing resources (cores) to the parallel tasks. This is done via an internal scheduling mechanism based on "work stealing" to distribute parallel work-items among "workers". Workers are a concept in Intel Cilk Plus similar to threads in other frameworks (for example, OpenMP). Because of the simplicity of the API and high degree of behind-the-scenes automation, Intel Cilk Plus can dramatically reduce the development workload and time while providing great performance. In the framework, we have only three keywords in Intel Cilk Plus: cilk_for, cilk_spawn and cilk_sync. Namely, in our study, we used cilk_for that enables programmers to parallelize C/C++ for loops.

However, note that this ease of use of Intel Cilk Plus functionality does not release the programmer from following the necessary precautions that apply to all parallelized loops. Avoiding issues such as race conditions is still the programmer's responsibility. In this case, Intel Cilk Plus provides C++ templates referred to as reducers in order to eliminate race conditions in parallel programs with certain patterns (see, e.g., [22]) [23]. In fact, Intel Cilk Plus is the vectorization and multi-threading capabilities are separable, and often the vectorization capabilities are useful in conjunction OpenMP. In our study, Intel Cilk Plus has particularly low cost "spawning", so it's a particularly good fit for highly recursive code where the base cases cannot be forced to be large chunks.

\subsection{OpenMP}

The OpenMP programming model provides an API with set of compiler directives, function calls, and environment variables that instruct the compiler how and where to use parallelism in the application. The directive based approach makes it possible to write sequentially consistent codes for easier maintenance. The well-known advantage of OpenMP is its global view of application memory address space that allows relatively fast development of parallel applications. OpenMP is supported by almost all major compilers [24].

The OpenMP can be used effectively for the parallelization of the originally sequential code because this requires only minimal code changes, and thus minimizing the logical mistakes of the programmer. The programmer does not create threads directly within the application code, but just inserts OpenMP directives for the compiler, which generates the code for threads during the translation. The advantage of this approach is obvious; in most cases, it is sufficient just to insert directives to indicate which section of code will running parallel. The original code does not need to be changed. The functionality of the originally sequenced code is clearly visible and the parallelization code is separable. Obviously, this approach is especially useful when it is necessary to parallelize the existing code.

In our study, we have used the following OpenMP directives valid for $\mathrm{C} / \mathrm{C}++$ programming languages:

- \#pragma omp parallel \{\} - defines a section of the parallel calculation,

- \#pragma omp for schedule (dynamic) - defines a parallel loop and each iteration will be assigned to threads dynamically.

The pragmas control how the program works. By the $\mathrm{C} / \mathrm{C}++$ standards, even if the compiler does not support pragmas, the program will run correctly, however, without parallelism. Therefore an adding the OpenMP directives can be done very safely. [25]

\subsection{Vectorization}

Used CPUs have different instruction vectorization sets. The HSW CPUs provide 256-bit vector registers and AVX/AVX2 (Advanced Vector Extensions) instruction sets. KNC provides 512-bit vector registers and IMCI instruction sets. The KNL nodes provide 512-bit vector registers and new AVX-512 instruction set. Intel compiler C/C++ language extension for array notations simplifies the code vectorization since it translates array notation any of targeted CPUs / instruction sets. However to get the most performance out of these processors, users need to take in account of CPU differences when applying vectorization instructions in their code, where the vector register size is the most significant parameter. In order to enable the compiler to generate efficient code for the assembly step, we have added _ restrict and const keywords to our computational kernels.

This is rather straightforward to do as the computational kernels are implemented using simple data structures and abstractions are only build on top of that layer. We have used the vectorization report of the Intel performance profiling tool (Intel VTune Amplifier) to check that the compiler has indeed sufficient information to vectorize the time intensive portions of our algorithm. Note, however, that the code for the KNL is essentially identical to the optimized code for all platforms.

In this article, all tests are run on the three machines with the following specifications: 
1) Intel multicore Platform Xeon Haswell-E (HSW): 6-core Intel Core i7-5930K series processor based on the Intel Xeon Haswell-E architecture with two thread per core; Frequency: 3.5 GHz; DDR4: 32GB; L1d cache: 32 kB; L1i cache: $32 \mathrm{kB}$; L2 Cache: $256 \mathrm{kB}$; L3 Cache: $15360 \mathrm{kB}$

2) Intel manycore Xeon Phi Knights Corner (KNC): a single 57-core Intel Xeon Phi coprocessor 3120A with four threads per core; Frequency: 1.1 GHz; GDDR5: 6 GB of RAM; L1d cache: $32 \mathrm{kB}$; L1i cache: $32 \mathrm{kB}$; L2 Cache: $28.5 \mathrm{MB}$

3) Intel manycore Xeon Phi Knights Landing (KNL): a single 68-core Intel(R) Xeon Phi(TM) CPU 7250 processor with four threads per core; Frequency: $1.4 \mathrm{GHz}$; DDR4: $96 \mathrm{~GB}$ of RAM with 16 GB MCDRAM memory at 1013.140 MHz; L1d cache: 32 kB; L1i cache: 32 kB; L2 Cache: 34 MB

The base scenario we used for most of our results sets $\delta=h=0.25$ and $\mathrm{N}+1=40$, corresponding to a ten-year term structure of quarterly rates. There are 3 possible swaptions with 5 possible maturities (lengths) per swaption and possible strike prices for the 3 swaptions. Amount price varies over time typically determined as a function of time to maturity in this example; however, it remains constant 0.2 values and number of simulations that Monte Carlo runs is 96000 . The application of Libor Rate Model (LRM) to estimating delta entails knowledge of the transition density of the underlying state variables. No such density is available in forward LIBOR models, so we use a Gaussian approximation using Intel Math Kernel Library (MKL).

\section{Performance Results}

The algorithm requires repeated simulations of chance that lend themselves well to parallel processing and vectorization. The simulations in this example are run in the following cases:

1. Serially code without any parallelization,

2. Array Notation (AN) for vectorization using Intel specific $\mathrm{C}++$ language extensions,

3. AN and Cilk implementation for task thread parallelism,

4. AN and OpenMP implementation for task thread parallelism.

To use target CPU architecture effectively, applications should effectively use VPU instructions on vector data, good locality of reference, and utilizes caches well in its core computations. However, the scheduling module (function cilkrts scheduler) of the Intel Cilk Plus runtime library is spent the majority of the CPU time on all platforms. It is beneficial to find a parallel scheduler with less overhead. For example, OpenMP framework is a better solution with less overhead and it is available for all processors (HSW, KNC, and KNL). The OpenMP standard provides similar functionality (tasking and dynamic number of threads in parallel regions), but in this application we were not able to achieve satisfactory performance results with OpenMP despite investing a greater development effort than we did with Intel Cilk Plus on HSW platform.

Table 1. shows the results for the single precision solution of the tiled. The performance is expressed in the computation time (run time). The first two trials for each data point were not taken into the statistical average.

\begin{tabular}{|c|c|c|c|}
\hline \multirow[b]{2}{*}{ Test (Monte Carlo) } & \multirow{2}{*}{$\begin{array}{c}\text { Intel Multi-core Platform } \\
\text { Haswell }(\mathrm{HSW}) \\
\end{array}$} & \multicolumn{2}{|c|}{ Intel Many Integrated Core Xeon Phi Architecture } \\
\hline & & Knights Corner (KNC) & Knights Landing (KNL) \\
\hline Performance time & \multicolumn{3}{|c|}{$[\mathrm{ms}]$} \\
\hline 1. Serial code & 1973 & 25964 & 6837 \\
\hline 2. AN & 275 & 1640 & 439 \\
\hline 3. AN + cilk for & 47 & 21 & 6 \\
\hline 4. $\mathrm{AN}+\mathrm{omp}$ for & 54 & 15 & 4 \\
\hline Recommended Customer Price $(\mathrm{RCP})^{1}$ & $\$ 594.00$ & $\$ 1,491.00$ & $\$ 2,436.00$ \\
\hline Thermal Design Power (TDP) ${ }^{1}$ & $140 \mathrm{~W}$ & $300 \mathrm{~W}$ & $215 \mathrm{~W}$ \\
\hline Theoretical Peak Performance (TPP) $^{1}$ & $384 \mathrm{GFLOP} / \mathrm{s}$ & $1.2 \mathrm{TFLOP} / \mathrm{s}$ & $3 \mathrm{TFLOP} / \mathrm{s}$ \\
\hline
\end{tabular}

Table 1. Timing results for used algorithm on targets CPU architectures

On all architectures, we have achieved the same result (average discounted payoff is 49.439308) based on equation (5) and base scenario for an estimation of the valuation of a portfolio of European LIBOR-based swaptions using a Monte Carlo simulation. Timing results in table 1. show that the KNC times with serial code has 13 times more execution time, with 2.5 times more price and over 2 times more power than the HSW. 
In below formulas we have evaluated the economic profitability and the energy efficiency using executing time (run time) in representative benchmarks.

$$
\begin{aligned}
& \text { Cost }_{H S W}=R C P * \text { Time }=594 * 47=27,918 \\
& \text { Cost }_{K N C}=R C P * \text { Time }=1,491 * 15=22,365 \\
& \text { Cost }_{K N L}=R C P * \text { Time }=2,436 * 4=9,774 \\
& \text { Dissipation }_{H S W}=T D P * \text { Time }=140 * 47=6,580 \\
& \text { Dissipation }_{K N C}=T D P * \text { Time }=300 * 15=4,500 \\
& \text { Dissipation }_{K N L}=T D P * \text { Time }=215 * 4=860
\end{aligned}
$$

Results show that the KNL with OpenMP evaluates payoff European LIBOR-based swaptions significantly faster (small the computation time), more economically profitable (8) (small the Cost factor) and more energy efficient (11) (small the Dissipation factor) than nearly comparable KNC and HSW.

\section{Conclusion}

In this paper, we evaluate the performance of such an optimization algorithm on modern accelerators (more specifically, the Intel Multi-core Platform Xeon Haswell, the Intel Many-core Xeon Phi Knights Corner and the Intel Many-core Xeon Phi Knights Landing). We present timing results for all codes and discuss the similarities and differences between the four implementations. Optimization and parallelization for the HSW, KNC and KNL code is done using the Intel Compiler v17.0 (vectorization) with Intel Cilk Plus and OpenMP frameworks. We've achieved same results (average discounted payoff is 49.439308) on all architectures.

This study shows that the KNL with OpenMP framework is significantly faster, more economic profitable and more energy efficient than KNC and the HSW. The results shows that the thread scheduling overhead in Intel Cilk Plus framework has a negative influence on the overall performance on all architectures, because this is done via an internal scheduling mechanism based on a "work stealing" to distribute parallel work-items among "workers". In OpenMP, we inserted directives, which defines a parallel loop and each iteration will be assigned to threads dynamically. Further, the study shows that KNC architecture has not significantly better results than HSW with regard to the price and the energy consumption.

In this way, KNL architecture have the best ways to compute, energy efficiency, economic profitability and achieve massive increases in performance with effectively used strong parallelization and vectorization (Cilk Plus and OpenMP) frameworks.

In further work, we are pursuing ways to further research/apply this, especially for newer targets that have different architectural characteristics.

\section{References}

[1] Ferreiro, A.M.; García, J.A.; López-Salas, J.G. \& Vázquez, C. (2014). SABR/LIBOR market models: pricing and calibration for some interest rate derivatives, Appl. Math. Comput. 242, 2014, pp. 65-89.

[2] Dupire, B. (1994). "Pricing with a smile," Risk 7 (1), 1994, pp. 18-20.

[3] Derman, E. \& Kani, I. (1994). Riding on a smile, Risk 7 (2), 1994, pp. 32-39.

[4] Andersen, L. B. G. \& Andreasen, J. (2000). Volatility Skews and Extensions of the Libor Market Model, Journal Applied Mathematical Finance, Volume 7, 2000, pp. 1-32

[5] Merton, R. C. (1976). Option pricing when underlying stock returns are discontinuous, Journal of Financial Economics, Volume 3, Issues 1-2, January-March 1976, pp. 125-144

[6] Eberlein, E.; Keller, U. \& Prause, K. (1998). New insights into smile, mispricing, and value at risk: the hyperbolic model, The Journal of Business, Volume 71, 1998, pp. 371-405

[7] Jamshidian, F. (2001). Libor market model with semimartigales, Available from: https://www.researchgate.net/publication/228750716_LIBOR_market_model_with_semimartingales, Accessed: 2017-06-04 
[8] Glasserman, P. \& Kou, S. (2003). The term structure of simple forward rates with jump risk, The Journal Mathematical Finance, Volume 13, Issue 3, 2003, pp. 345-444.

[9] Glasserman, P. \& Merener, N. (2003). Numerical solution of jump-diffusion LIBOR market models, The Journal Finance and Stochastics, 2003, pp. 1-27.

[10] Belomestny, D. \& Schoenmakers, J.G.M. (2011). A jump-diffusion Libor model and its robust calibration, Quantitative Finance, Volume 11, 2011, pp. 529-546.

[11]Eberlein, E. \& Ozkan, F. (2005). The Lévy LIBOR model, The Journal Finance and Stochastics, 2005, pp. 327348.

[12] Giles, M.B. \& Glasserman, P. (2006). Smoking adjoints: fast Monte Carlo Greeks, RISK, 2006.

[13] Giles, M. (2007). Monte Carlo evaluation of sensitivities in computational finance, Tech. Rep. NA07/12, 2007.

[14] Glasserman, P. (2003). Monte Carlo Methods in Financial Engineering, Springer-Verlag, New York.

[15]Finance: Monte Carlo (2013). Available from: https://software.intel.com/en-us/code-samples/intel-ccompiler/application-domains/finance/Monte-Carlo, Accessed: 2017-06-04

[16] Glasserman, P. \& Zhao, X. (2000). Arbitrage-free discretization of lognormal forward, Finance and Stochastics, Volume 4, Issue 1, 2000, pp. 35-68.

[17] Spicar, R. \& Januska, M. (2015). Use of Monte Carlo Modified Markov Chains in Capacity Planning. Procedia Engineering, vol. 100, pp. 953-959.

[18] Jamshidian, F. (1997). Libor and swap rate models, Finance Stochast. 1, 1997, pp. 293-330.

[19]Keegan, S. (2008). Vibrato Monte Carlo and the calculation of greeks, Available from: http://eprints.maths.ox.ac.uk/716/1/Thesis_SK.pdf, Accessed: 2017-06-04

[20] Intel Cilk Plus, (2017). Available from: https://software.intel.com/en-us/intel-cilk-plus, Accessed: 2017-06-04

[21] Cilk home page, (2017). Available from: http://www.cilkplus.org/, Accessed: 2017-06-04

[22] McCool, M.; Reinders, J. \& Robison, A. (2012). Structured parallel programming: patterns for efficient computation, Elsevier, 2012, ISBN: 978-0-12-415993-8

[23] Asai, R. \& Vladimirov, A. (2015). Intel Cilk Plus for complex parallel algorithms: Enormous Fast Fourier Transforms (EFFT) library, Parallel Computing, Volume 48, 2015, pp. 125-142.

[24] Akhter, S. \& Roberts, J., (2006). Multi-Core Programming: Increasing Performance through Software Multithreading. $1^{\text {st }}$ Edn., Intel Press, ISBN: 13: 978-0976483243, pp: 360.

[25]Hofierka, J.; Lacko, M. \& Zubal, S. (2016). Parallelization of interpolation, solar radiation and water flow simulation modules in GRASS GIS using OpenMP, Computers \& Geosciences, Volume 107, October 2017, pp. $20-27$ 